\title{
Controlled fracture of the medial wall versus structural autograft with bulk femoral head to increase cup coverage by host bone for total hip arthroplasty in osteoarthritis secondary to developmental dysplasia of the hip: a retrospective cohort study
}

\author{
Ping Mou' ${ }^{1}$ Kai Liao ${ }^{2}$, Hui-lin $\mathrm{Chen}^{3}$ and Jing Yang ${ }^{1^{*}}$
}

\begin{abstract}
Background: Many methods have been proposed to increase cup coverage by host bone during primary total hip arthroplasty (THA) in hip osteoarthritis secondary to developmental dysplasia of the hip (DDH). However, there was no study comparing the results of controlled fracture of the medial wall with a structural autograft with a bulk femoral head.

Methods: Sixty-seven hips classified as Crowe II/III were retrospectively included in this cohort study, which consisted of 33 controlled fractures (group A) and 34 structural autografts (group B). The Harris Hip Scores (HHS) were recorded. The radiological assessments were analyzed. Also, complications are assessed. The paired-sample $t$ test was used for data analysis before and after the operation, while the independent sample $T$ test was used for the comparison between the two groups. The Pearson chi-square test or the Fisher exact test was used to analyze the qualitative comparative parameters. Kaplan-Meier was utilized in the analysis of survivorship with the end points as a revision for any component. Results: All patients were reconstructed acetabulum at the anatomical location. HHS increased greatly for both groups $(p=0.18)$. No statistic difference was observed for the two groups in postoperative leg-length discrepancy $(0.51 \pm 0.29$ $\mathrm{cm}$ for group $A$ and $0.46 \pm 0.39 \mathrm{~cm}$ for group $B, p=0.64)$, postoperative height of the hip center $(2.25 \pm 0.42 \mathrm{~cm}$ for group $A$ and $2.09 \pm 0.31 \mathrm{~cm}$ for group $B, p=0.13$ ), and inclination of the cup ( $39 \pm 4^{\circ}$ for group $A$ and $38 \pm 3^{\circ}$ for group $B, p=0.65)$. The rate of cup coverage for group B $(94 \pm 2 \%)$ was better than for group $A(91 \pm 5 \%),(p=.009)$. The rate of cup protrusio was $48 \pm 4 \%$ for group $A$. For both groups, no statistical difference was observed in the cup diameter $(p>.05)$, while group A showed less operation time than group B $(p<.001)$. No complications were observed at the latest follow-up.

(Continued on next page)
\end{abstract}

\footnotetext{
* Correspondence: cd_yangjing@163.com

'Department of Orthopedic Surgery, West China Hospital, West China

Medical School, Sichuan University, \#37 Guoxue Road, Chengdu 610041,

Sichuan Province, People's Republic of China

Full list of author information is available at the end of the article
}

C C The Author(s). 2020 Open Access This article is licensed under a Creative Commons Attribution 4.0 International License, which permits use, sharing, adaptation, distribution and reproduction in any medium or format, as long as you give appropriate credit to the original author(s) and the source, provide a link to the Creative Commons licence, and indicate if changes were made. The images or other third party material in this article are included in the article's Creative Commons licence, unless indicated otherwise in a credit line to the material. If material is not included in the article's Creative Commons licence and your intended use is not permitted by statutory regulation or exceeds the permitted use, you will need to obtain permission directly from the copyright holder. To view a copy of this licence, visit http://creativecommons.org/licenses/by/4.0/ The Creative Commons Public Domain Dedication waiver (http://creativecommons.org/publicdomain/zero/1.0/) applies to the data made available in this article, unless otherwise stated in a credit line to the data. 
(Continued from previous page)

Conclusion: Controlled fracture of the medial wall to increase cup coverage by host bone at the anatomical location can act as an alternative technique for DDH Crowe II/III with the advantage of shorter operation time and less technically demanding.

Keywords: Total hip arthroplasty, Developmental dysplasia of the hip, Cup coverage, Controlled fracture of the medial wall, Structural autograft

\section{Introduction}

Total hip arthroplasty (THA) in osteoarthritis secondary to developmental dysplasia of the hip (DDH) is challenging [1]. And many studies [2-4] have reported unfavorable clinical outcomes and higher rate of complications of THA in osteoarthritis secondary to DDH compared with primary hip osteoarthritis. Because DDH presents a spectrum of anatomical disorders including the femur and the acetabulum, the abnormal femoral change of the oversize anteversion and narrow medullary cavity can be easily handled by a modular hip stem, which can correct the overanteverted femoral neck and provide rotational stability if subtrochanteric shortening osteotomy was performed [5]. For patients without excessive femoral anteversion and narrow medullary cavity, monobloc stem can act as an alternative technique. And many studies have reported similar results [6-8]. But for the distorted acetabulum especially DDH Crowe II/III, it always manifests with pathomorphologic changes including shallow true acetabulum, formation of a neoacetabulum, and superolateral bony deficiency $[1,9-$ 11], all of which make it more complicated and technically demanding to balance acetabular reconstruction at the anatomical location and rate of cup coverage by host bone $[12,13]$.

There are three main techniques to increase cup coverage by host bone in primary THA: creation of a high hip center $[14,15]$, structural autograft with bulk femoral head [16, 17], and medial protrusio technique $[13,18]$. Although the creation of a high hip center can simplify the process of acetabulum management and has been widely used for revision acetabular reconstruction, yet many scholars $[13,17,19]$ agreed on inserting the acetabular cup into the anatomical hip center due to superior biomechanics, better fixation, and stability. Structural bulk bone grafting is another effective technique suggested by scholars to increase cup coverage by host bone $[17,20]$. Bone grafting can restore bone mass and realize a superior rate of cup coverage by superolateral fixation of the processed autologous femoral head. But the complexity of operation techniques and potential risks including resorption or collapse of the graft and aseptic loosening keep it away from us [21]. Medial protrusio technique consisting of medial wall penetration, medial wall osteotomy, and controlled fracture of the medial wall is a series of methods that deepens the acetabulum and insert the acetabular cup with a medial aspect beyond the Kohler' line to achieve a higher rate of cup coverage. With the technique, bone grafting is not necessary to increase the rate of cup coverage. Zhang et al. [13] and Hartofilakidis et al. [18] have published excellent outcomes and safety of the technique. But currently, there are studies just reporting the clinical efficacy of bulk bone autograft or medial protrusio technique. And no cohort study was published. To our knowledge, this was the first study comparing the results of controlled fracture of the medial wall with structural bone grafting to increase cup coverage by host bone for hip osteoarthritis secondary to DDH Crowe II/III.

We hypothesize that for hip osteoarthritis secondary to DDH Crowe II/III, controlled fracture of the medial wall presents similar results like structural autograft with bulk femoral head to increase cup coverage by host bone at the anatomical location on clinical measurements and radiological evaluations.

\section{Material and methods Study design}

The study is a retrospective cohort study performed through the retrieval of information on a hospital information system from January 2007 to December 2014. And the targeted patients were recalled to accomplish the follow-up. Study approval was obtained from the Clinical Trials and Biomedical Ethics Committee of West China Hospital.

\section{Patient selection criteria}

The following are the inclusion criteria: (1) patients diagnosed with end-stage hip osteoarthritis secondary to DDH and scheduled to undergo primary THAs during the target period, (2) classification of DDH belonging to type II or III according to Crowe [2], and (3) patients performed controlled fracture of the medial wall or structural autograft with bulk femoral head to increase cup coverage by host bone by the same senior surgeon (the corresponding author). The following are the exclusion criteria: (1) patients performed THAs due to other reasons, (2) other types of DDH according to Crowe, (3) increasing cup coverage by other methods, (4) THA 
performed by other senior surgeons, and (5) patients lost to follow-up.

Ultimately, we selected and analyzed the data of the patients performed by controlled fracture of the medial wall (group A) or structural autograft with a bulk femoral head (group B). All analyzed participants were identified as Crowe II/III. And we called up the patients to return to our hospital to complete the follow-up. So, we obtained the latest clinical and radiological data.

\section{Surgical techniques}

All acetabular cups were placed at the true acetabulum. After general anesthesia, all patients were located in a lateral position, the hips were exposed with a posterolateral approach, and then sawing off the femoral heads and resecting the osteophytes and synovium around the joint as well as the soft tissue in the cotyloid notch of the true acetabulum were performed. The acetabular preparation is conducted by standardized reaming (approximately $45^{\circ}$ of abduction and $15^{\circ}$ of anteversion). And the deepening was continued until the outer surface of the internal pelvic cortex was reached. The acetabulum trial component was then inserted into the acetabulum and placed at the appropriate abduction and anterversion to examine the rate of cup coverage. If the rate was not satisfactory or the initial stability of the cup was not realized, we would perform a controlled fracture of the medial wall or structural autograft with the processed bulk femoral head to increase the rate of cup coverage. The surgical option selected to increase cup coverage was determined by the senior surgeon at the time of surgery based on careful planning on AP radiograph and the degree of anatomical deformity. The senior surgeon made the final decision according to preoperative assessments and clinical experience intraoperatively.

The detailed steps of controlled fracture of the medial wall were described as follows: Firstly, the medial wall was spherically fractured by osteotome with the center in the top of the cotyloid notch and one-third diameter of the anteroposterior dimension of the true acetabulum. Take care not to perforate the internal layer of the periosteum. Secondly, migrate the superior autogenous mud-like cancellous graft to the fractured area and disperse uniformly. Thirdly, the cementless acetabular component was placed with appropriate orientation and pressure. At last, before placing the liner, examine the initial stability of the component. If the stability was not satisfactory, supplemental screws would be used to reinforce the early cup stability.

The detailed steps of structural autograft with bulk femoral head were described like Kim and Kadowaki
[17]. In brief, firstly, the cancellous surface of the resected bulk femoral head was prepared, and the femoral head was shaped to accommodate bone deficiency. Secondly, the pseudoacetabular floor was reamed to expose the cancellous bone. Thirdly, both cancellous surfaces were impacted with screws to prevent micromotion between the graft and the host bone. At last, insert the cementless acetabular component and check the stability of the component.

For the management of the femur, after expanding the medullary cavity routinely, place the trial component and femoral head. Then, try to realize hip joint reduction and check out the stability on different directions and assess sciatic nerve tension by palpation. For the condition that it was difficult to realize hip joint reduction or excessive tension of the sciatic nerve, we would perform transverse subtrochanteric shortening osteotomy. Then, after finishing the procedures, we would evaluate the range of motion (ROM) of the hip, limb length, and nerve tension. Once all of these were satisfactory, we finally placed the components. Ultimately, irrigated the articular cavity, placed a drainage if transverse subtrochanteric shortening osteotomy was performed, and sutured the incision.

\section{Perioperative regimen}

For all patients, positive motion exercises were initiated on the bed after recovering from anesthesia. Prophylactic intravenous antibiotics were used within the first $24 \mathrm{~h}$ postoperatively. Additionally, low-molecular-weight heparin (LMWH) and painkillers were systematically managed to prevent deep venous thrombosis (DVT) and relieve pain, respectively. The drainage tube was removed within $24 \mathrm{~h}$.

From the first postoperative day on, all of the patients were allowed to partial weight-bearing exercises with the help of walker aid and full weight-bearing exercises after 6 weeks. Once the patients can realize independent walking, the walker aid can be removed. For the ones receiving transverse subtrochanteric shortening osteotomy, internal and external rotations of the hip were forbidden until the bony union of the osteotomy.

\section{Clinical measurements}

Clinical details were recorded including operation time, cup diameter, ROM of the hip, and Harris Hip Scores (HHS) [22]. The operation time was defined as the time from skin incision to skin suturing. ROM consisting of flexion, extension, and abduction and Harris scores were examined by 2 authors. Postoperative HHS (the total score is 100) are defined as excellent (>90), good (80-89), fair (70-79), and poor $(<70)$. 


\section{Radiological assessments}

Standard AP radiograph was obtained before and after surgery. Preoperatively and postoperatively, radiographs were analyzed by 2 authors. The assessments included leg-length discrepancy (LLD), the height of the hip center, inclination of the cup, rate of the cup coverage, and rate of the medial protrusion. LLD was assessed by the standardized-trochanteric method to avoid the influence of pelvic and femoral inclination on the radiographs [23]. The standardized-trochanteric method requires the vertical distance from the inter-teardrop line to the center of rotation and the femoral vertical distance (center of rotation to the lesser trochanter) reference to the femoral anatomical axis. So, the unilateral distance is defined as the difference of the two vertical distances. And LLD is equal to the difference of the two unilateral distances (Fig. 1). The height of the hip center is defined as the perpendicular distance from the femoral head center to the inter-teardrop line [24]. The inclination of the cup is defined as the angle between a horizontal line joining the ischial spines and a line parallel to the opening plane of the cup [25]. The rate of cup coverage and the rate of medial protrusion are measured according to the methods introduced by Dorr et al. [26] and Kim et al. [27]. The rate of the cup coverage is defined as the ratio of the degree of the cup covered by the host bone and $180^{\circ}$ (Fig. 2). The rate of the medial protrusion is defined as the ratio of the degree of cup medialization beyond the Kohler's line and $180^{\circ}$ (Fig. 2).

\section{Complications}

The complications are recorded including early-onset and late-onset complications during the period of perioperation and follow-up. The early-onset ones consist of infection, intraoperative fracture, DVT, pulmonary embolism, and nerve palsy. Meanwhile, the late-onset ones consist of postoperative dislocation, nonunion of the femoral osteotomy, graft collapse, polyethylene wear, osteolysis, and aseptic loosening [28-30].

\section{Statistical analysis}

Statistical analysis was performed using the SPSS software for Windows version 22.0 (SPSS, Chicago, IL). The level of statistical significance was set at $p<0.05$. The results were expressed as the mean \pm standard deviation. The paired-sample $t$ test was used for data analysis before and after the operation, while the independent sample $T$ test was used for the comparison between the two groups. The Pearson chi-square test or the Fisher exact test was used to analyze the qualitative comparative parameters. Kaplan-Meier was utilized in the analysis of survivorship with the end points as a revision for any component.

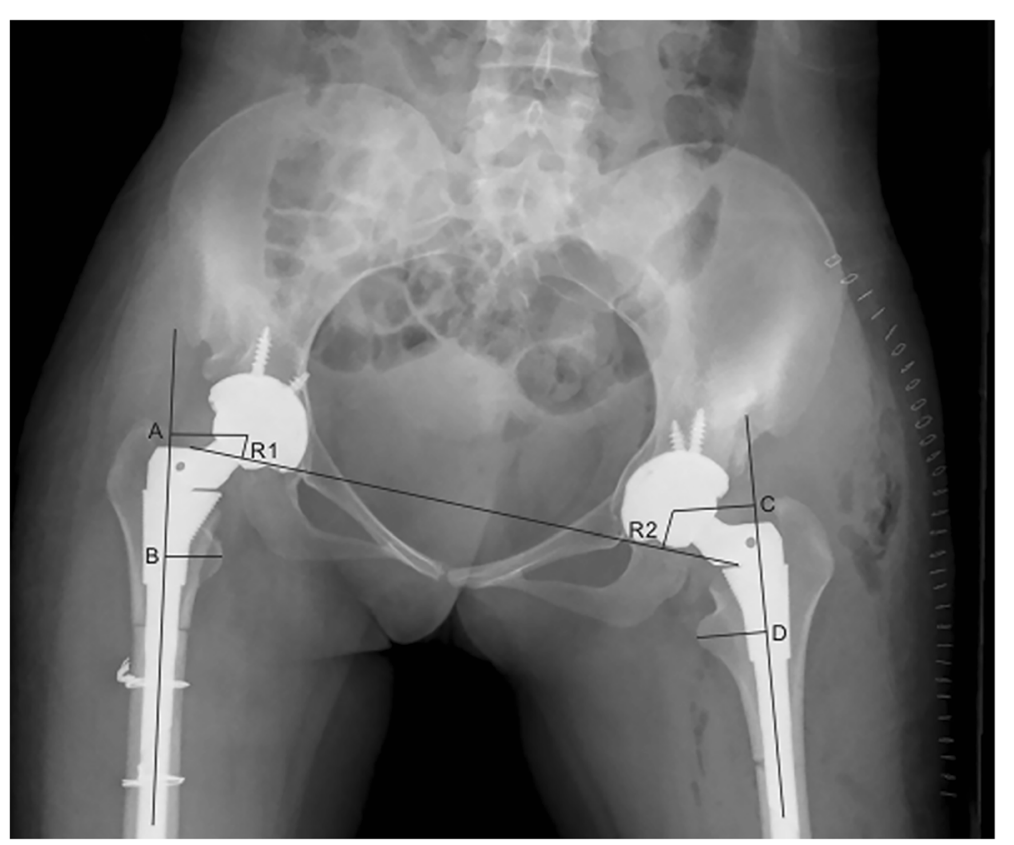

Fig. 1 Diagram showing a standardized-trochanteric method to assess leg-length discrepancy. R1 and R2 are the vertical distance from the bilateral center of rotation to the inter-teardrop line. Line $A B$ and line $C D$ are the anatomical axes of the femurs. Point $A$ and point $C$ are the perpendicular intersections from the center of rotation to the femoral anatomical axis. Point $B$ and point $D$ are the perpendicular intersections from the tip of the lesser trochanter to the femoral anatomical axis. $\mathrm{H} 1$ and $\mathrm{H} 2$ are equal to $A B$ and $C D$, respectively. Leg-length discrepancy = $(H 1-R 1)-(H 2-R 2)$ 


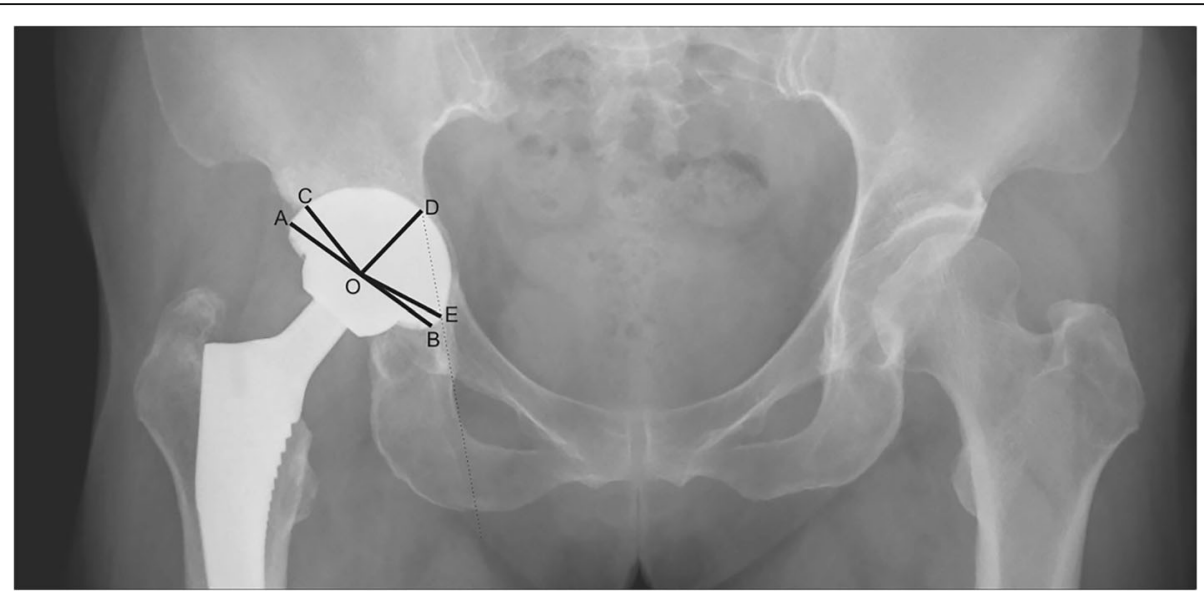

Fig. 2 Schematic showing the measurement of medial protrusion and cup coverage. Point $O$ is the center of the cup component. $A B$ is the diameter of the cup component. Point $C$ is the intersection between the edge of the cup implant and the ilium. Point $D$ and point $E$ are the intersections between the medial edge of the acetabular component and Kohler's line. The rate of cup coverage $=\left[\left(180^{\circ}-\angle A O C\right) / 180^{\circ}\right] \times 100 \%$. The rate of medial protrusion $=\left(\angle \mathrm{DOE} / 180^{\circ}\right) \times 100 \%$

\section{Results}

For group A, the mean age of all patients (male:female = 4:27) was 49.2 years (49-67), and the mean body mass index (BMI) was $22.8 \mathrm{~kg} / \mathrm{m}^{2}$ (17.2-27.4). Besides, the patients (33 hips) were analyzed with 12 hips classified as type II and 21 hips classified as type III according to Crowe. For group B, the mean age of all patients (male: female $=6: 24)$ was 50.9 years (33-63), and the mean BMI was $22.9 \mathrm{~kg} / \mathrm{m}^{2}$ (17.3-29.7). Moreover, the patients (34 hips) were analyzed with 10 hips classified as type II and 24 hips classified as type III according to Crowe. These patients underwent 67 THAs performed by the same senior surgeon. Five patients of group A and 6 patients of group B were performed transverse

Table 1 Baseline of all recruited patients

\begin{tabular}{|c|c|c|}
\hline DDH (no. = 67 hips) & Group A (no. = 33 hips) & Group B (no. = 34 hips) \\
\hline Mean age (years) & $49.2 \pm 8.3($ range, 49-67) & $50.9 \pm 9.1$ (range, 63-33) \\
\hline Male:female & $4: 27$ & $6: 24$ \\
\hline Mean height $(\mathrm{cm})$ & $157.4 \pm 6.2($ range, $149-175)$ & $155.0 \pm 6.5($ range, $145-170)$ \\
\hline Mean weight (kg) & $56.3 \pm 6.7$ (range, 43-70) & $54.8 \pm 7.6$ (range, 43-68) \\
\hline Mean BMI $\left(\mathrm{kg} / \mathrm{m}^{2}\right)$ & $22.8 \pm 2.6($ range, $17.2-27.4)$ & $22.9 \pm 3.3($ range, 17.3-29.7) \\
\hline \multicolumn{3}{|l|}{ Crowe classification } \\
\hline$\|$ & 12 & 10 \\
\hline III & 21 & 24 \\
\hline Follow-up (months) & $85 \pm 36$ & $78 \pm 35$ \\
\hline \multicolumn{3}{|l|}{ Cup type } \\
\hline Pinnacle & 33 & 34 \\
\hline \multicolumn{3}{|l|}{ Stem type } \\
\hline Corail & 15 & 10 \\
\hline Tri-lock & 3 & 3 \\
\hline S-rom & 15 & 21 \\
\hline \multicolumn{3}{|l|}{ Friction couples } \\
\hline Ceramic-on-ceramic & 28 & 30 \\
\hline Ceramic-on-polyethylene & 5 & 4 \\
\hline \multicolumn{3}{|l|}{ Subtrochanteric osteotomy } \\
\hline Yes/no & $5 / 28$ & $6 / 28$ \\
\hline
\end{tabular}


Table 2 Range of motion and Harris Hip Scores for all recruited patients preoperatively and postoperatively of both two groups

\begin{tabular}{|c|c|c|c|c|c|c|c|c|}
\hline \multirow[t]{2}{*}{$\mathrm{DDH}$} & \multicolumn{2}{|c|}{ Group A } & \multicolumn{2}{|l|}{ Group B } & \multicolumn{2}{|l|}{ Intra } & \multicolumn{2}{|l|}{ Inter } \\
\hline & Pre & Post & Pre & Post & Group A & Group B & Pre & Post \\
\hline Flexion $\left(^{\circ}\right)$ & $88 \pm 25$ & $113 \pm 7$ & $91 \pm 16$ & $116 \pm 5$ & $t=-5.86, p<.001^{*}$ & $t=-9.97, p<.001^{*}$ & $T=-0.55, p=.59$ & $T=-1.37, p=.18$ \\
\hline Extension $\left({ }^{\circ}\right)$ & $-1 \pm 3$ & $0 \pm 0$ & $-2 \pm 3$ & $0 \pm 0$ & $t=-1.75, p=.095$ & $t=-2.30, p=.031^{*}$ & $T=0.26, p=.54$ & - \\
\hline Abduction $\left({ }^{\circ}\right)$ & $20 \pm 11$ & $38 \pm 4$ & $23 \pm 6$ & $36 \pm 3$ & $t=-10.29, p<.001^{*}$ & $t=-12.88, p<.001^{*}$ & $T=-1.08, p=.29$ & $T=1.58, p=.12$ \\
\hline HHS & $38 \pm 6$ & $87 \pm 6$ & $40 \pm 4$ & $89 \pm 6$ & $t=-65.35, p<.001^{*}$ & $t=-77.36, p<.001^{*}$ & $T=-1.24, p=.22$ & $T=-1.36, p=.18$ \\
\hline
\end{tabular}

Pre Preoperatively, Post Postoperatively, HHS Harris Hip Scores, Intra Intra-group comparisons, Inter Inter-group comparisons, DDH Developmental dysplasia of the hip

${ }^{*} p$ values with statistical significance

subtrochanteric shortening osteotomy. All patients were followed up using a standard clinical and radiographic protocol mentioned above. Also, we recorded the information about the components. And the related details were presented in Table 1. All implants of both groups used during the procedures were from DePuy, Warsaw, IN. The patients requiring THAs on both hips were performed separately.

\section{Clinical outcomes}

Ultimately, 61 patients (33 hips of group A and 34 hips of group B) were followed up for $85 \pm 36$ months for group $\mathrm{A}$ and $78 \pm 35$ months for group $\mathrm{B}$. All patients were satisfied with the results that the pain had decreased and gait had improved markedly compared with preoperative status. For group A, the average HHS improved from $38 \pm 6$ points preoperatively to $87 \pm 6$ points at the latest follow-up. According to the postoperative HHS, 15 hips $(45.5 \%)$ are defined as excellent, 13 hips (39.4\%) good, and 5 hips (15.2\%) fair. The average flexion, extension, and abduction of the hip increased from $88 \pm 25^{\circ},-1 \pm 3^{\circ}$, and $20 \pm 11^{\circ}$ preoperatively to $113 \pm 7^{\circ}, 0 \pm 0^{\circ}$, and $38 \pm 4^{\circ}$ at the final follow-up, respectively. For group B, comparably, the average HHS improved from $40 \pm 4$ preoperatively to $89 \pm 6$ at the latest follow-up. According to the postoperative HHS, 17 hips (50\%) are defined as excellent, 12 hips (35.3\%) good, and 5 hips (14.7\%) fair. The average flexion, extension, and abduction of the hip increased from $91 \pm 16^{\circ},-2 \pm$ $3^{\circ}$, and $23 \pm 6^{\circ}$ preoperatively to $116 \pm 5^{\circ}, 0 \pm 0^{\circ}$, and 36 $\pm 3^{\circ}$ at the latest follow-up, respectively (Table 2). The mean operation time of both groups showed a statistical difference $(T=-6.49, p<.001)$. Group A was 76.8 (51125) min, while group $B$ was $107.2(80-143)$ min (Table $3)$. The outer and inner diameters of the cup in group A were $46(44-50)$ and $29.7(28-36) \mathrm{mm}$, while that of group B were 46.5 (44-52) and $29.5(28-36) \mathrm{mm}$, respectively, both of which showed no statistical difference $(p>.05)$ (Table 3).

\section{Radiological outcomes}

For group A, at the latest follow-up, LLD restored from $2.31 \pm 1.65 \mathrm{~cm}$ preoperatively to $0.51 \pm 0.29 \mathrm{~cm}$. The height of the hip center was restored from $4.34 \pm 1.03$ to $2.25 \pm 0.42 \mathrm{~cm}$. The inclination of the cup was $39 \pm 4^{\circ}$. The rate of the cup coverage was $91 \pm 5 \%$. And the rate of the cup protrusio was $48 \pm 4 \%$ (Tables 3 and 4) (Fig. 3). Additionally, for group B, at the final follow-up, LLD restored from $2.46 \pm 1.37$ to $0.46 \pm 0.39 \mathrm{~cm}$. The height of the hip center restored from $4.59 \pm 0.59$ to $2.09 \pm 0.31$ $\mathrm{cm}$. the inclination of the cup was $38 \pm 3^{\circ}$. The rate of the cup coverage was $94 \pm 2 \%$ (Tables 3 and 4) (Fig. 4). For the patients performed transverse subtrochanteric shortening osteotomy of both two groups, most of them (10 of 11) realized a bony union at 6 months after surgery, and the remaining one realized at 9 months postoperatively. From the X-ray films of the latest follow-up, we did not find radiolucent lines, visible implant loosening, and periprosthetic osteolysis for both groups. Moreover, we observed no patient encountering graft collapse or progressive migration of the implant from the radiographs.

Table 3 Comparison of radiographic outcomes postoperatively and clinical outcomes of all included patients

\begin{tabular}{llll}
\hline DDH & Group A & Group B & Inter \\
\hline IC $\left(^{\circ}\right)$ & $39 \pm 4^{\circ}$ & $38 \pm 3^{\circ}$ & $T=0.46, p=.65$ \\
RCC (\%) & $91 \pm 5 \%$ & $94 \pm 2 \%$ & $T=-2.78, p=.009^{*}$ \\
Outer diameter of the cup (mm) & $46.8 \pm 2.0$ & $46.5 \pm 1.8$ & $p=.111$ \\
Inner diameter of the cup (mm) & $29.7 \pm 2.2$ & $29.5 \pm 2.2$ & $p=.94$ \\
Operation time (min) & $76.8 \pm 20.9$ & $107.2 \pm 17.2$ & $T=-6.49, p<.001^{*}$ \\
\hline
\end{tabular}

IC Inclination of cup, RCC Rate of cup coverage, Inter Inter-group comparisons, DDH Developmental dysplasia of the hip

${ }^{*} p$ values with statistical significance 
Table 4 Comparison of leg-length discrepancy and height of the hip center by radiography preoperatively and postoperatively of all included patients

\begin{tabular}{|c|c|c|c|c|c|c|c|c|}
\hline \multirow[t]{2}{*}{$\mathrm{DDH}$} & \multicolumn{2}{|l|}{ Group A } & \multicolumn{2}{|l|}{ Group B } & \multicolumn{2}{|l|}{ Intra } & \multicolumn{2}{|l|}{ Inter } \\
\hline & Pre & Post & Pre & Post & Group A & Group B & Pre & Post \\
\hline LLD (cm) & $2.31 \pm 1.65$ & $0.51 \pm 0.29$ & $2.46 \pm 1.37$ & $0.46 \pm 0.39$ & $t=4.69, p<.001^{*}$ & $t=6.52, p<.001^{*}$ & $T=-0.32, p=.75$ & $T=0.48, p=.64$ \\
\hline $\mathrm{HHC}(\mathrm{cm})$ & $4.34 \pm 1.03$ & $2.25 \pm 0.42$ & $4.59 \pm 0.59$ & $2.09 \pm 0.31$ & $t=8.66, p<.001^{*}$ & $t=22.56, p<.001^{*}$ & $T=-1.02, p=.31$ & $T=1.54, p=.13$ \\
\hline
\end{tabular}

Pre Preoperatively, Post postoperatively, Intra Intra-group comparisons, Inter Inter-group comparisons, LLD Leg-length discrepancy, HHC Height of the hip center, $D D H$ Developmental dysplasia of the hip ${ }^{*} p$ values with statistical significance

\section{Complications}

None of the patients suffered from early-onset complications. But 2 hips of group A and 2 hips of group B (3 hips classified as Crowe III and 1 hip classified as Crowe II) encountered intraoperative fracture of the proximal femur, which were addressed well by wires. For the lateonset complications such as dislocation, osteolysis and radiolucent lines were not observed regardless of the surgical methods and Crowe classification. However, 1 hip classified as Crowe III from group B performed transverse subtrochanteric shortening osteotomy suffered from delay union and finally achieved bony union at 9 months postoperatively. The latest radiograph of the patient showed excellent stability of the acetabular cup and stem. No revision of all patients was required during the follow-up period, although there were apparent differences in bone stock and upper migration between Crowe II and III. And the survival rate of the prosthesis
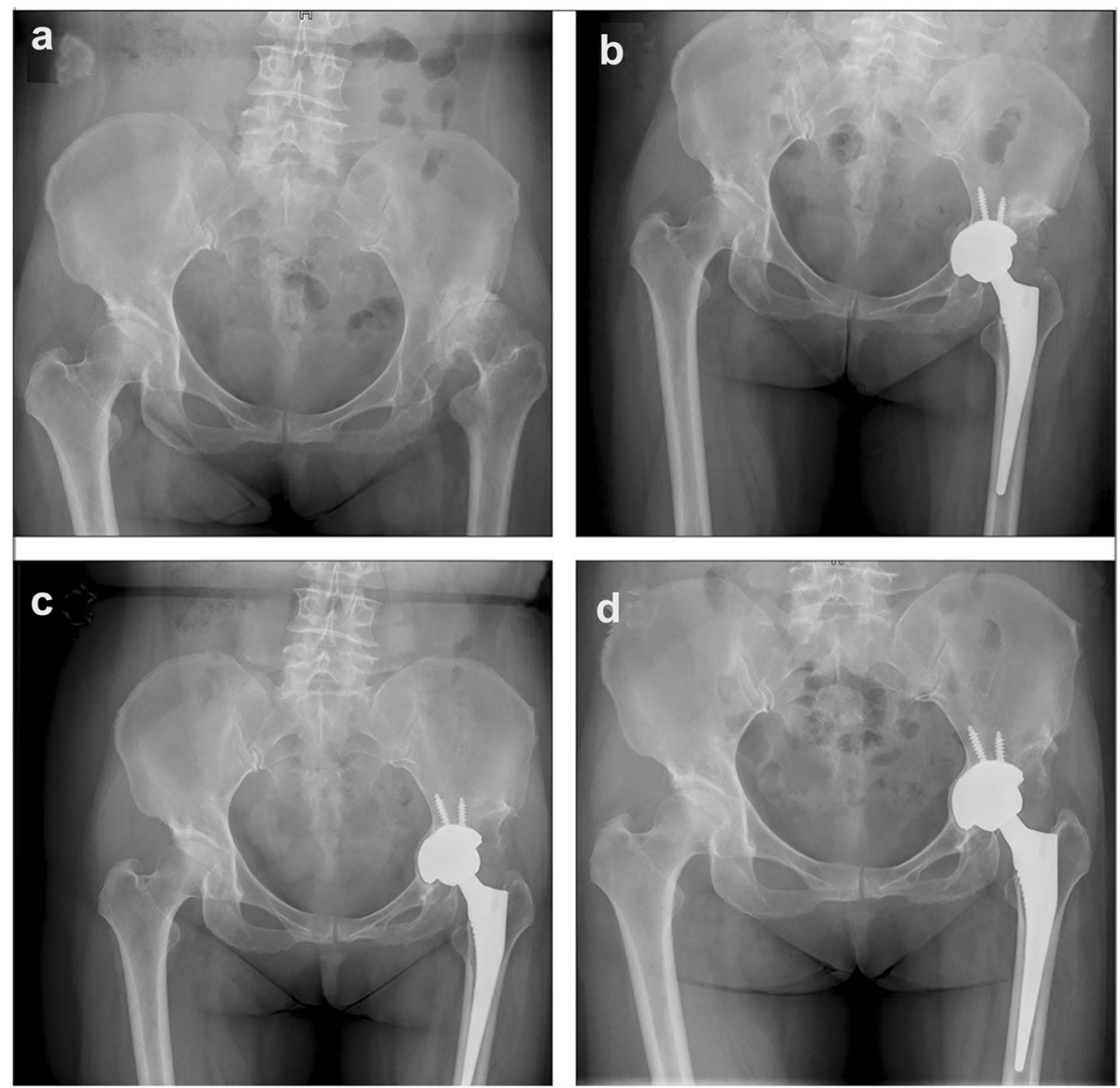

Fig. 3 Case presentation of controlled fracture of the medial wall for THA. a A 54-year-old woman was diagnosed with DDH Crowe III on the radiograph of the pelvis preoperatively. $\mathbf{b}$ The radiograph of the pelvis after surgery immediately showed the rate of cup coverage was $86 \%$, the rate of medial protrusion was $52 \%$, and the leg-length discrepancy was $0.38 \mathrm{~cm}$. c The radiograph of the pelvis at 1-year follow-up showed the medial wall was a bony union and no aseptic loosening of the component. $\mathbf{d}$ The radiograph of the pelvis at 76-month follow-up showed no aseptic loosening and migration of the component 

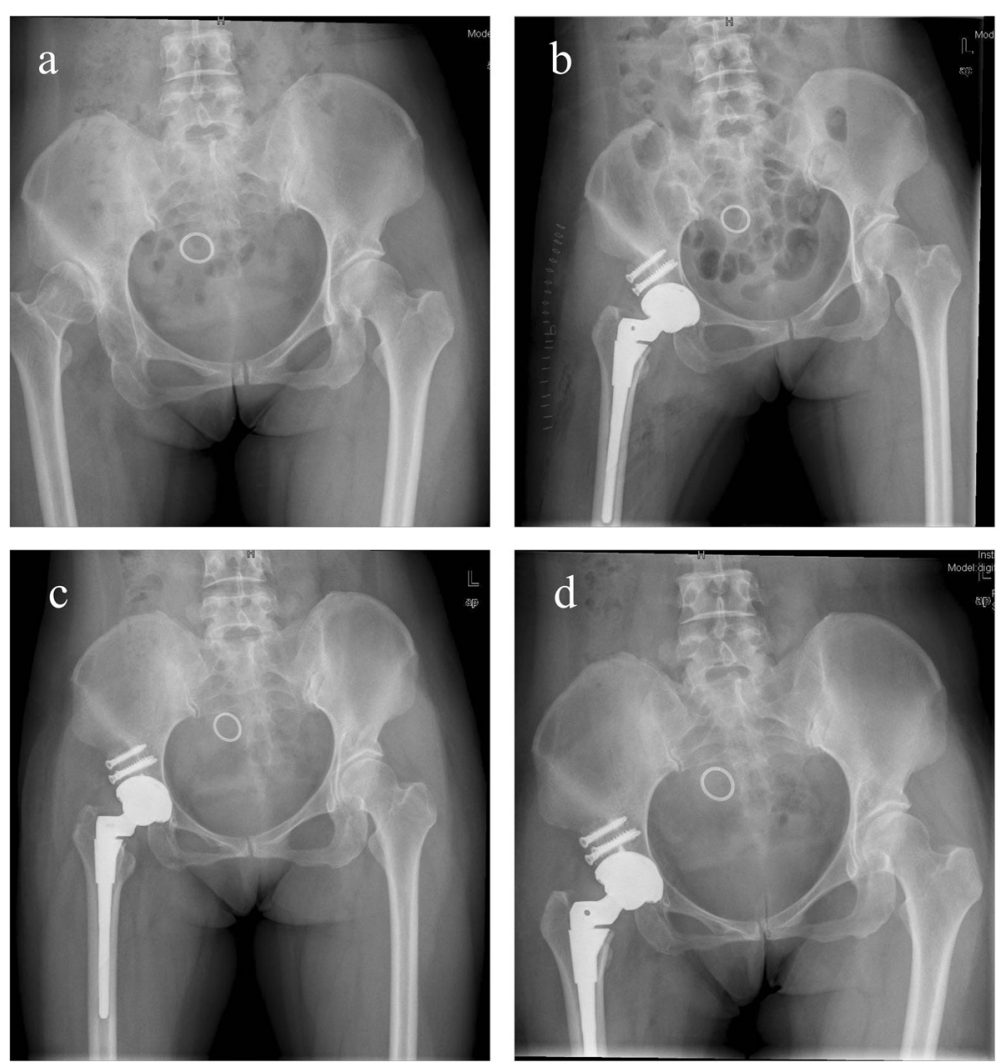

Fig. 4 Case presentation of structural autograft with a bulk femoral head for THA. a A 51-year-old woman was diagnosed with DDH Crowe III on the radiograph of the pelvis preoperatively. $\mathbf{b}$ The radiograph of the pelvis after surgery immediately showed the rate of cup coverage was $95 \%$ and the leg-length discrepancy was $0.16 \mathrm{~cm}$. $\mathbf{c}$ The radiograph of the pelvis at 1-year follow-up showed the interface between the graft and the host bone had been obscure and no aseptic loosening of the component. $\mathbf{d}$ The radiograph of the pelvis at 84-month follow-up showed incorporation of grafted bone and no aseptic loosening and migration of the component

was $100 \%$ regardless of the surgical methods and Crowe classification.

\section{Discussion}

The most important finding of this study was that the clinical measurements and radiological assessments of both two groups almost restore to normal, and no one showed complications at the latest follow-up. Besides, for the patients, the pain has relieved and the gait has improved as well as no complaining about self-perceived LLD. So, both two approaches are effective to increase cup coverage by host bone and reconstruct acetabulum. According to the current literature [10], because of abnormal anatomy, secondary osteoarthritis of DDH occurs at a relatively young age (an average of 42 years old), and for the younger population, the long-term survival of implant was reported to be lower than that of the general ones due to more activity [31, 32]. Besides, for DDH Crowe II/III, the biggest challenge lies in cup coverage and acetabular reconstruction. Also, the study [33] has demonstrated that the rate of failure of the acetabular component showed a positive correlation with the severity of DDH. So, the surgeons are more concerned about how to obtain adequate initial stability of the cup in order to realize long-term survival of the acetabular component and postpone potential revision surgery.

There is no consensus on the position of the acetabular component. And the placement of the acetabular implant can be located in the true acetabulum or high hip center. The high hip center is defined as the perpendicular distance from the femoral head center to the interteardrop line more than $35 \mathrm{~mm}$ [24]. Some scholars [11, $13,17,19,34]$ agreed on inserting the cup into the true acetabulum due to superior biomechanics, better fixation, and more bone mass. Placing the implant at the anatomical center of the hip enables optimal abductor muscle function, and the bone mass for fixation of the component is larger than at a more proximal level [12]. However, we must address problems such as limb lengthening [12], nerve palsy [12], and less coverage of the cup [19]. Gratifyingly, subtrochanteric osteotomy [10], structural autograft [17], and medial protrusio technique [11] have provided novel and valid methods. 
Additionally, the creation of a high hip center was also proposed for primary acetabular reconstruction and was reported to have good long-term outcomes and showed no difference in polyethylene wear $[15,35]$. Nevertheless, there are many other problems to face. Firstly, high acetabular reconstruction often results in high, lateral, and oversized cup placement leading to the problems like fixation, primary stability, and restoration of normal hip biomechanics [10]. Secondly, at this high level, the bone stock is insufficient, and shearing forces on the acetabular component may lead to early loosening. Meanwhile, a longer lever arm for body weight can result in excessive load to the hip joint $[19,36]$. Thirdly, longer prosthetic neck length used to balance leg lengths possibly leads to neck-liner impingement [13]. Lastly, the patients performed primary THA owing to DDH usually are younger than hip osteoarthritis, and most of them likely need revision surgery, which may be more difficult owing to limited bone stock [17]. Bicanic et al. [37] reported that every millimeter of lateral displacement of the acetabular cup compared with the ideal rotation center resulted in an increase of $0.7 \%$ in hip load and every millimeter of proximal displacement an increase of $0.1 \%$ in hip load. This accounts for a high rate of failure if the cup component is placed in a high hip center. Additionally, Chen et al. [14] and Stans et al. [38] have demonstrated that using a high hip center during acetabular reconstruction in DDH patients had a higher failure rate. So, based on these reasons, we have chosen anatomic placement of the acetabular cup. And the results of this study were that LLD was approximately $5 \mathrm{~mm}$ and no one complained about self-perceived LLD, if we reconstructed the acetabulum at the true location. Also, aseptic loosening was not found at the latest follow-up. These results demonstrated the anatomic reconstruction of the acetabulum was a superior choice once again.
Currently, the literatures $[18,39]$ have proved the medial protrusio technique was an effective method to increase the rate of the cup coverage without a bulk femoral head autograft. The medial protrusio technique includes controlled medial wall fracture, medial wall osteotomy, and medial wall penetration [11]. And the reasons why we chose controlled medial wall fracture as a cotyloplasty are the simplification and safety compared with the other two techniques. Besides, the current literatures [18, 39] have demonstrated that the long-term survival of THA performing controlled fracture of the medial wall showed no difference compared with that of general THA at 10 years. For medial wall osteotomy, it is a more technically demanding process, and the thickness of the medial wall should be not less than $10 \mathrm{~mm}$ according to Zhang et al. [13]. For medial wall penetration, the loss of bone stock is more than the medial wall fracture. And it is easy to ream excessively leading to the damage of the acetabular rim. We have provided an illustration (Fig. 5) of the comparison of three medial protrusion techniques. And we hope better explanations can be realized for the differences. To our knowledge, there is no report for more than 10 years about the medial wall osteotomy and medial wall penetration demonstrating comparable outcomes with controlled medial wall fracture. So, based on these factors, we thought controlled medial wall fracture is a better one for increasing cup coverage in THA with DDH.

Sufficient initial stability of cementless cup is imperative for successful osseointegration and good long-term survival, but medial protrusion may decrease this stability. So, what rate of medial protrusion of the cup is the best? Dorr et al. [26] recommended this rate should be less than 45\%, and Kim et al. [27] suggested it should be a

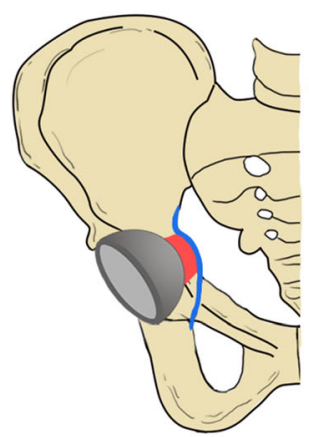

$\mathrm{b}$

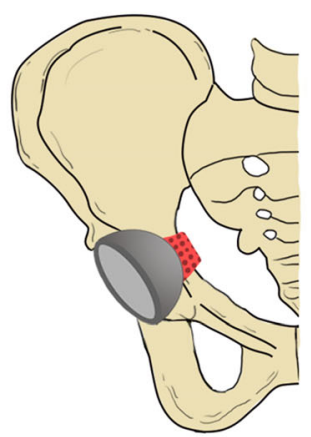

c

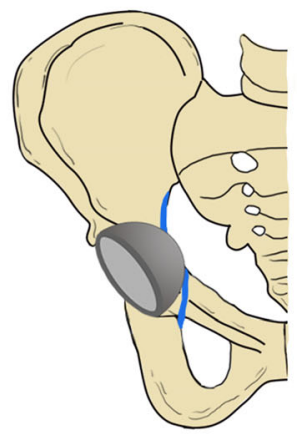

Fig. 5 Diagram showing the differences between the three medial protrusion techniques increasing the rate of cup coverage. a The illustration of a medial wall fracture. The blue color showed the complete internal layer of the periosteum. The red color showed the autogeneous mud-like cancellous graft and fractured medial wall. b The illustration of medial wall osteotomy. The red color showed the medially displaced bone block c The illustration of medial wall penetration. The medial aspect of the acetabular cup beyond the Kohler's line with a discontinuous internal layer of the periosteum colored in blue and bone loss 
within 50-60\%. Besides, according to Zha et al. [11], a prospective 6- to 9-year follow-up of 43 consecutive patients using the medial protrusio technique in cementless THA for DDH demonstrated that the rate of medial protrusion more than $60 \%$ had a high aseptic loosening rate in the midterm. Also, the excessive rate of medial protrusion may possibly result in cup component migration into the pelvis. And the rate of medial protrusion of our study was $48 \pm 4 \%$, and no complications were observed at the final follow-up. The purpose of medial protrusion was to increase cup coverage by host bone and realize initial cup stability. So, we considered it was reasonable to realize enough cup coverage with the least rate of the medial protrusion. According to the published studies and the results of our research, we suggested that the rate of medial protrusion should be less than $60 \%$. If needed, supplementary screws can be used for initial cup stability. Additionally, the amount of protrusion was evaluated by preoperative templating on an AP radiograph. Also, the surgeon would conduct assessment again intraoperatively after standard acetabular reaming. Preoperative planning would provide a reference for the intraoperative amount of medial protrusion. And the operators can judge the amount of medial protrusion according to intraoperative fluoroscopy compared with preoperative planning. The final amount of medial protrusion was determined on the preoperative planning and intraoperative evaluation.

For patients diagnosed with $\mathrm{DDH}$, the abnormal femoral morphologies contribute to the complexity of the cases, which include excessive femoral anteversion, coxa valga, and small diaphyseal diameters [10]. For these cases, modular stem-like SROM stem (Depuy, Warsaw, IN) may be needed. Compared with monobloc stem, modular stem provides intraoperative flexibility in femoral reconstruction. Sleeves with various height and width options can be chosen to accommodate different metaphyseal morphologies. Also, the different combinations of the neck and stem can optimize offsets, avoid LLD, and match different medullary cavity [40, 41]. If subtrochanteric shortening osteotomy was performed, modular stem-like SROM stem can provide rotational stability [5]. In brief, modular stem possesses advantages including accommodating abnormal femoral medullary cavity, optimizing offset, avoiding LLD, and providing rotational stability. However, there are concerns associated with modular stem including junction failure and corrosion due to the slippage and micromotion that occurred at the stem-sleeve interface [38, 42, 43]. The study from Bobyn et al. demonstrated that the wear particle was not significant enough to cause osteolysis and loosening [44]. Also, Seufert and McGrory [43] and Kong et al. [45] have reported modular stem can successfully accommodate the distorted anatomy of the proximal femur and achieved optimal stem version as well as excellent clinical outcomes. So, modular stem can be recommended as an alternative choice to reconstruct the femora in THA for patients diagnosed with DDH.

The technique, controlled fracture of medial wall, has several advantages to deal with unsatisfying cup coverage compared with structural autograft with a bulk femoral head. Firstly, it can simplify the operation and does not ask for special surgical instruments. Meanwhile, this technique does not prolong the operation time and cause obviously additional damage to the patients. Secondly, the area of operated medial wall belongs to the bony union because of the application of autogenous mud-like cancellous graft and protection of the internal layer of the periosteum. And the integrity of the acetabular rim does not damaged. So, there is little influence on the primary stability and bone ingrowth of the cup component. Thirdly, medialization of the cup component can increase the rate of the cup coverage and decrease wear due to the increase of the abductor lever arm and decreased loading of the hip joint. However, the primary concern of this technique is possibly excessive medial protrusio resulting in unsatisfied primary stability of the cup component or disastrous migration of the cup into pelvis when postoperative weight-bearing exercises. Besides, the range of medial fracture is not easy to control. And The future revision arthroplasty in the patients operated with controlled fracture of the medial wall is another concern, especially the higher fixation problem and bone deficiency. For the mentioned concerns, we suggest that firstly do not chase for an excessive rate of medial protrusio if the cup component can realize enough rate of cup coverage and initial stability. Secondly, the application of autogenous mud-like cancellous graft and protection of the internal layer of the periosteum play a vital role in the bony union of the medial wall. Postoperative function exercise should be rational and not too be ambitious. Thirdly, the manmade fracture of the medial wall could realize bony union by application of autogenous mud-like cancellous graft and protection of the internal layer of the periosteum. So, no excessive bone loss was compared with primary THA. Additionally, with the development of material science, we believe many other effective methods can handle the fixation problem and bone deficiency easily.

The limitations of the current study were the retrospective research with small population and relatively short period of follow-up, and we could not acknowledge the long-term outcomes. Additionally, when we conducted radiological assessments, we just used 2dimensional images to accomplish evaluations, which might compromise the robustness of the final results. 
But, it was the first study to compare the effectiveness of controlled fracture of the medial wall with that of structural bone grafting to increase cup coverage and reconstruct acetabulum. And the study demonstrated that a controlled fracture of the medial wall could increase the rate of cup coverage without technically demanding, which can act as a selectable method to increase cup coverage and reconstruct acetabulum.

\section{Conclusion}

Controlled fracture of the medial wall is an effective and safe technique to increase the rate of cup coverage for THA in osteoarthritis secondary to DDH Crowe II/III. With the advantage of less technical demand, we recommended it as an alternative technique to increase cup coverage by host bone for THA in hip osteoarthritis secondary to DDH Crowe II/III.

\section{Abbreviations}

THA: Total hip arthroplasty; DDH: Developmental dysplasia of the hip; ROM: Range of motion; LMWH: Low-molecular-weight heparin; DVT: Deep venous thrombosis; HHS: Harris hip scores; LLD: Leg-length discrepancy

\section{Acknowledgements}

We thank all the patients for participating in our study, and we are grateful to the staff of our department for their support and contribution to this study. We would also like to thank Dr. Ke Xiao from the Department of Orthopaedic Surgery, West China Hospital, for helping with the drawing of the schematic diagram in Fig. 5.

\section{Authors' contributions}

PM and $\mathrm{KL}$ designed this study. $\mathrm{KL}$ and HLC were responsible for gathering, analyzing, and interpreting the data. MP wrote the manuscript. JY made contributions to revising the manuscript for crucial intellectual content. JY contributed to the revision of the manuscript and interpretation of the data. The final version of the text has been reviewed and approved by all authors. $\mathrm{PM}$ and $\mathrm{KL}$ contributed equally to this work and should be considered as equal first authors.

\section{Funding}

This research was funded by the National Natural Science Foundation of China Program (81601936) and the Science and Technology Planning Project of Sichuan Province (2019YFS0266).

\section{Availability of data and materials}

The datasets used and/or analyzed during the current study are available from the corresponding author on reasonable request.

\section{Ethics approval and consent to participate}

This study has been approved by the Clinical Trials and Biomedical Ethics Committee of West China Hospital, Sichuan University. Each author certifies that all investigations were conducted in accordance with the ethical principles. All participants involved in the study gave their informed consent.

\section{Consent for publication}

All data collected in this study have consent for publication.

\section{Competing interests}

The authors announce that they do not have any competing interests.

\section{Author details}

'Department of Orthopedic Surgery, West China Hospital, West China Medical School, Sichuan University, \#37 Guoxue Road, Chengdu 610041, Sichuan Province, People's Republic of China. ${ }^{2}$ Department of Radiology, West China Hospital, West China Medical School, Sichuan University, Chengdu 610041, Sichuan Province, People's Republic of China. ${ }^{3}$ Clinical
Medicine, West China Hospital, West China Medical School, Sichuan University, Chengdu 610041, Sichuan Province, People's Republic of China.

Received: 22 July 2020 Accepted: 10 November 2020

Published online: 26 November 2020

\section{References}

1. Gustke K. The dysplastic hip: not for the shallow surgeon. Bone Joint J. 2013;95-B(11 Suppl A):31-6.

2. Crowe JF, Mani VJ, Ranawat CS. Total hip replacement in congenital dislocation and dysplasia of the hip. J Bone Joint Surg Am. 1979;61:15-23.

3. Hartofilakidis G, Karachalios T, Stamos KG. Epidemiology, demographics and natural history of congenital hip disease in adults. Orthopedics. 2000;23: $823-7$.

4. Garvin KL, Bowen MK, Salvati EA, Ranawat CS. Long-term results of total hip arthroplasty in congenital dislocation and dysplasia of the hip: a follow-up note. J Bone Joint Surg Am. 1991;73:1348-54.

5. Tamegai H, Otani T, Fujii H, Kawaguchi Y, Hayama T, Marumo K. A modified S-ROM stem in primary total hip arthroplasty for developmental dysplasia of the hip. J Arthroplasty. 2013:28:1741-5.

6. Shi X-T, Li C-F, Cheng C-M, Feng C-Y, Li S-X, Liu J-G. Preoperative planning for total hip arthroplasty for neglected developmental dysplasia of the hip. Orthop Surg. 2019;11(3):348-55.

7. Lee KH, Jo WL, Ha YC, Lee YK, Goodman SB, Koo KH. Total hip arthroplasty using a monobloc cementless femoral stem for patients with childhood Perthes' disease. Bone Joint J. 2017:99-B(4):440-4.

8. Luo ZY, Wang HY, Wang D, Pan H, Pei FX, Zhou ZK. Monobloc implants in cementless total hip arthroplasty in patients with Legg-Calve-Perthes disease: a long-term follow-up. BMC Musculoskelet Disord. 2017;18(1):386.

9. Hartofilakidis G, Stamos K, loannidis TT. Low friction arthroplasty for old untreated congenital dislocation of the hip. J Bone Joint Surg Br. 1988;70: 182-6.

10. Greber EM, Pelt CE, Gililland JM, Anderson MB, Erickson JA, Peters CL. Challenges in total hip arthroplasty in the setting of developmental dysplasia of the hip. J Arthroplasty. 2017;32:S38-44.

11. Zha GC, Sun JY, Guo KJ, Zhao FC, Pang Y, Zheng X. Medial protrusio technique in cementless total hip arthroplasty for developmental dysplasia of the hip: a prospective 6- to 9-year follow-up of 43 consecutive patients. J Arthroplasty. 2016;31:1761-6.

12. Kosuge D, Yamada N, Azegami S, Achan P, Ramachandran M. Management of developmental dysplasia of the hip in young adults: current concepts. Bone Joint J. 2013;95:732-7.

13. Zhang $H$, Huang $Y$, Zhou YX, Zhou YX, Lv M, Jiang ZH. Acetabular medial wall displacement osteotomy in total hip arthroplasty: a technique to optimize the acetabular reconstruction in acetabular dysplasia. J Arthroplasty. 2005;20:562-7.

14. Chen M, Luo ZL, Wu KR, Zhang XQ, Ling XD, Shang XF. Cementless total hip arthroplasty with a high hip center for Hartofilakidis type $B$ developmental dysplasia of the hip: results of midterm follow-up. J Arthroplasty. 2016;31:1027-34.

15. Kaneuji A, Sugimori T, Ichiseki T, Yamada K, Fukui K, Matsumoto T. Minimum ten-year results of a porous acetabular component for Crowe I to III hip dysplasia using an elevated hip center. J Arthroplasty. 2009;24:187-94.

16. Harris WH, Crothers $\mathrm{O}$, Oh J. Total hip replacement and femoral-head bonegrafting for severe acetabular deficiency in adults. J Bone Joint Surg Am. 1977:59:752-9.

17. Kim M, Kadowaki T. High long-term survival of bulk femoral head autograft for acetabular reconstruction in cementless THA for developmental hip dysplasia. Clin Orthop Relat Res. 2010;468:1611-20.

18. Hartofilakidis G, Georgiades G, Babis GC, Yiannakopoulos CK. Evaluation of two surgical techniques for acetabular reconstruction in total hip replacement for congenital hip disease: results after a minimum ten-year follow-up. J Bone Joint Surg Br. 2008;90:724-30.

19. Hartofilakidis $G$, Karachalios T. Total hip arthroplasty for congenital hip disease. J Bone Joint Surg Am. 2004;86:242-50.

20. de Jong PT, Haverkamp D, van der Vis HM, Marti RK. Total hip replacement with a superolateral bone graft for osteoarthritis secondary to dysplasia: a long-term follow-up. J Bone Joint Surg Br. 2006;88:173-8.

21. Zahar A, Papik K, Lakatos J, Cross MB. Total hip arthroplasty with acetabular reconstruction using a bulk autograft for patients with developmental 
dysplasia of the hip results in high loosening rates at mid-term follow-up. Int Orthop. 2014;38:947-51.

22. Harris WH. Traumatic arthritis of the hip after dislocation and acetabular fractures: treatment by mold arthroplasty. An end-result study using a new method of result evaluation. J Bone Joint Surg Am. 1969;51:737-55.

23. Keršič $M$, Dolinar $D$, Antolič $V$, Mavčič $B$. The impact of leg length discrepancy on clinical outcome of total hip arthroplasty: comparison of four measurement methods. J Arthroplasty. 2014;29:137-41.

24. Li H, Mao Y, Oni JK, Dai K, Zhu Z. Total hip replacement for developmental dysplasia of the hip with more than $30 \%$ lateral uncoverage of uncemented acetabular components. Bone Joint J. 2015;95:1178-83.

25. Mu W, Yang D, Xu B, Mamtimin A, Guo W, Cao L. Midterm outcome of cementless total hip arthroplasty in Crowe IV-Hartofilakidis type III developmental dysplasia of the hip. J Arthroplasty. 2016;31:668-75.

26. Dorr LD, Tawakkol S, Moorthy M, Long W, Wan Z. Medial protrusio technique for placement of a porous-coated, hemispherical acetabular component without cement in a total hip arthroplasty in patients who have acetabular dysplasia. J Bone Joint Surg Am. 1999;81:83-92.

27. Kim YL, Nam KW, Yoo JJ, Kim YM, Kim HJ. Cotyloplasty in cementless total hip arthroplasty for an insufficient acetabulum. Clin Orthop Surg. 2010;2:148-53.

28. Bernasek TL, Haidukewych GJ, Gustke KA, Hill O, Levering M. Total hip arthroplasty requiring subtrochanteric osteotomy for developmental hip dysplasia: 5- to 14-year results. J Arthroplasty. 2007:22:145-50.

29. Krych AJ, Howard JL, Trousdale RT, Cabanela ME, Berry DJ. Total hip arthroplasty with shortening subtrochanteric osteotomy in Crowe type-IV developmental dysplasia: surgical technique. J Bone Joint Surg Am. 2010; 92(Suppl 1 Pt 2):176-87.

30. Shinar AA, Harris WH. Bulk structural autogenous grafts and allografts for reconstruction of the acetabulum in total hip arthroplasty. Sixteen-yearaverage follow-up. J Bone Joint Surg Am. 1997;79:159-68.

31. Haraguchi A, Nakashima Y, Miyahara H, Esaki Y, Okazaki K, Fukushi Jl, et al. Minimum 10-year results of cementless total hip arthroplasty in patients with rheumatoid arthritis. Mod Rheumatol. 2017;27:598-04.

32. Tsukanaka M, Halvorsen V, Nordsletten L, EngesæTer IØ, EngesæTer LB, Marie Fenstad A, et al. Implant survival and radiographic outcome of total hip replacement in patients less than 20 years old. Acta Orthop. 2016;87:479-84.

33. Chougle A, Hemmady MV, Hodgkinson JP. Severity of hip dysplasia and loosening of the socket in cemented total hip replacement. A long-term follow-up. J Bone Joint Surg Br. 2005;87:16-20.

34. Pagnano W, Hanssen AD, Lewallen DG, Shaughnessy WJ. The effect of superior placement of the acetabular component on the rate of loosening after total hip arthroplasty. J Bone Joint Surg Am. 1996;78:1004-14.

35. Russotti GM, Harris WH. Proximal placement of the acetabular component in total hip arthroplasty. A long-term follow-up study. J Bone Joint Surg Am. 1991;73:587-92.

36. Bicanic G, Barbaric K, Bohacek I, Aljinovic A, Delimar D. Current concept in dysplastic hip arthroplasty: techniques for acetabular and femoral reconstruction. World J Orthop. 2014;5:412-24.

37. Bicanic G, Delimar D, Delimar M, Pecina M. Influence of the acetabular cup position on hip load during arthroplasty in hip dysplasia. Int Orthop. 2009; 33:397-02.

38. Stans AA, Pagnano MW, Shaughnessy WJ, Hanssen AD. Results of total hip arthroplasty for Crowe type III developmental hip dysplasia. Clin Orthop Relat Res. 1998;348:149-57.

39. Karachalios T, Roidis N, Lampropoulou-Adamidou K, Hartofilakidis G. Acetabular reconstruction in patients with low and high dislocation: 20- to 32-year survival of an impaction grafting technique (named cotyloplasty). Bone Joint J. 2013;95:887-92.

40. Gorski JM. Modular noncemented total hip arthroplasty for congenital dislocation of the hip. Case report and design rationale. Clin Orthop Relat Res. 1988:228:110-6.

41. Cameron HU. The two- to six-year results with a proximally modular noncemented total hip replacement used in hip revisions. Clin Orthop Relat Res. 1994:298:47-53.

42. Al-Khateeb H, Kwok IH, Hanna SA, Sewell MD, Hashemi-Nejad A. Custom cementless THA in patients with Legg-Calve-Perthes disease. J Arthroplasty. 2014:29:792-6.

43. Seufert CR, McGrory BJ. Treatment of arthritis associated with Legg-CalvePerthes disease with modular total hip arthroplasty. J Arthroplasty. 2015;30: $1743-6$
44. Bobyn JD, Tanzer M, Krygier JJ, Dujovne AR, Brooks CE. Concerns with modularity in total hip arthroplasty. Clin Orthop Relat Res. 1994;298:27-36.

45. Kong X, Sun Y, Yang M, Zhou Y, Chen J, Chai W, Wang Y. Total hip arthroplasty with modular stem for Crowe I and II developmental dysplasia of the hip. J Orthop Surg Res. 2019;14(1):362.

\section{Publisher's Note}

Springer Nature remains neutral with regard to jurisdictional claims in published maps and institutional affiliations.
Ready to submit your research? Choose BMC and benefit from:

- fast, convenient online submission

- thorough peer review by experienced researchers in your field

- rapid publication on acceptance

- support for research data, including large and complex data types

- gold Open Access which fosters wider collaboration and increased citations

- maximum visibility for your research: over $100 \mathrm{M}$ website views per year

At BMC, research is always in progress.

Learn more biomedcentral.com/submissions 